Provision has been made in the Navy Estimates for the observation of the total solar eclipse of 1905 August 30 by a party of three observers on the coast of Tunis, where the weather conditions are promising. It is proposed to take photographs of the corona for detail and extension with the Thompson 9-inch coronagraph, the 13-inch astrographic refractor, and the 4 -inch Dallmeyer rapid rectilinear lens, and also photographs of the spectrum with the two spectroscopes lent by Major Hills, R.E., as in rgoo and $190 \mathrm{I}$.

\section{THE OPTICAL CONVENTION.}

THE optical convention has just concluded a very successful meeting extending over four days; the exhibition and the papers attracted numerous visitors from all parts of the country. The papers led to much valuable discussion. An account of the exhibition and the president's address appeared in last week's NATURE.

The first group of papers dealt with the design of optical and scientific instruments. The Gauss theory was entrusted to Mr. Conrad Beck, who considered the theory of the equivalent planes of complete optical instruments; he dealt more particularly, with the complete microscope in relation to its " working distance."

Dr. Drysdale gave a general account of the aberrations of lens systems, submitting a classification and specification of the various aberrations to obtain an expression of opinion from those working at the subject. Mr. Chalmers gave a graphical method of representing the results of calculations of lens systems, and a modification of the Hartmann system of testing to permit of measuring and expressing aberrations in exactly the same form. This should make it possible to obtain the relation between the definition and the measured or calculated aberrations In the discussion Mr. Carson pointed out the importance of the relative intensity of the image disc and the aberration patch in estimating the performance of lenses.

Mr. Walter Rosenhain criticised the mechanical design of certain types of instruments; he showed that, in many cases, the ideals of the instrument maker were in conflict with sound engineering principles, and suggested directions in which improvements might be looked for.

Diffraction in optical instruments was discussed by $\mathrm{Mr}$. J. W. Gordon in an important theoretical paper; his conclusions, which would modify many of our ideas on optical systems, are now being submitted to a definite experimental test.

A group of papers related to interference phenomena. Mr. J. Rheinberg exhibited a method of producing achromatic interference bands which is likely to have numerous applications. Mr. Stansfield described a simple form of Michelson interferometer specially suitable for demonstration. Prof. Watkin and Mr. Morrow exhibited their apparatus for calibrating extensometers by observing the displacement of interference bands.

Mr. Twyman described the manufacture of the Echelon spectroscope, stating the accuracy required in the plates and the precautions used to obtain it. This apparatus was exhibited and compared with the Lummer "parallel plate " arrangement for obtaining resolution of spectrum lines. Mr. Blakesley discussed the various forms of prism which could be used in constant deviation spectroscopes and some of their applications. Mr. Newall dealt with astronomical "spectroscopes, demonstrating that the limits of usefulness of the present type of spectroscope were almost reached in the case of faint stars, as the intensity of light necessary for photographing their spectra can only be obtained at the sacrifice of the purity of the spectrum or the certainty of identification of the lines, and that no very marked improvement is likely to be obtained from the use of larger objectives, on account of the increased absorption in the prisms required. He suggested the use of gratings.

Lord Rayleigh dealt with the subject of polish, pointing out the distinction between the process of grinding, which consists of the removal of comparatively large flakes, and that of polishing, which he regards as molecular, the rough nesses of the surface being reduced to dimensions smaller than the wave-length of light. Experiments on the thick ness of glass removed by polishing and by etching with hydrofluoric acid under various conditions were illustrated. In the discussion $\mathrm{Mr}$. Walter Rosenhain cited evidence to show that the surface flow which has been recognised in the polishing of metals also occurs in glass.

Mr. Walter Rosenhain dealt with the possibilities of progress in optical glass; he described the limitations to the production of vitreous fluxes of extreme properties, and advanced the view that media of widely different optical properties could only be obtained by the production of large homogeneous crystals. Physicochemical considerations were cited to indicate lines upon which this difficult problem might be solved.

A number of instruments for optical measurements were described, Prof. Poynting exhibiting his form of parallel plate micrometer. Mr. Blakesley described his apparatus for the measurement of focal length of lenses, with applications to other optical measurements. Mr. Chalmers described a new form of refractometer for obtaining the refractive index of glass in lens form. The lens is inserted in a trough containing a suitable transparent liquid, and the difference of the refractive indices is deduced from the approximate curvatures of the lens and its power in the liquid, with an accuracy comparable with that of the best refractometers.

Mr. Baugh described the use of invar tapes for baseline measurements.

Dr. Drysdale discussed the requirements of small telescopes and binoculars, with special reference to the field of view and illumination of the image. He indicated the method he had employed in calculations for prism binoculars, showing how he had been led to use glass of high refractive index for the prisms. He described a special form of photometer for determining the absorption in binoculars.

Mr. A. C. Jolley gave a critical review of photometric standards and apparatus; he described a modification of the Violle platinum standard, and discussed the difficult problems connected with heterochromatic photometry; his results indicate that the accuracy claimed by Sir W. Abney is far too high, especially when readings by different observers are compared. He concludes that a discrimination photometer is the most trustworthy instrument for comparing different colours.

Mr. Milne exhibited his new form of spectrophotometer. The apparatus is especially suitable for determinations of the absorption of light of specified wave-length by liquids.'

$\mathrm{Mr}$. Bull discussed the theory of tricolour filters, plates, and inks. He concluded that it was most satisfactory to adjust each independently of errors in the adjustment of the others. The filters should have a certain amount of overlap, the colour of the overlap of two filters being the colour of the printing ink corresponding to the other filter.

$\mathrm{Mr}$. Crawley discussed the limits of stereoscopic vision; the resuits of his measurements point to a much greater accuracy in judging distances by stereoscopic effect than is generally admitted.

Mr. H. L. Taylor discussed the effects of astigmatism on the accommodation of the eye. Two new forms of ophthalmometer were demonstrated, one being the EttlesCurties, which is valuable for the perfection of its mechanical adjustments, and the use of complementary colours for the mires; it is so arranged that the corneal microscope can be readily attached. The ophthalmometer shown by Mr. Sutcliffe contains a number of variations from ordinary forms; the mire is an almost complete ring illuminated by a special lamp, and the method of doubling the image is novel.

Dr. Walmsley gave an account of the attempts which have been made to provide technical education for those engaged in the optical industry, and the existing facilities; he outlined the scheme for the establishment of a British Institute of Technical Optics. The convention decided to memorialise the London County Council to support the scheme.

Major-General Waterhouse gave an account of the history of telephotography.

In the evening lecture, Prof. Silvanus P. Thompson gave a most interesting account of the various forms of Nicol's prism and its modern equivalents.

$$
\text { NO. } 1858 \text {, VOL. } 72]
$$

